\title{
Influencia del consumo de soja sobre la masa ósea
}

de Luis DA, Pérez Castrillón JL, Aller R, Culebras J. Influencia del consumo de soja sobre la masa ósea. An Med Interna (Madrid) 2007; 24: 361-364.

\section{INTRODUCCIÓN}

La soja es un producto alimenticio de moda en los últimos tiempos debido a las propiedades beneficiosas que se le han atribuido sobre distintos trastornos, especialmente ligados a la menopausia. Uno de los efectos atribuidos a la soja es su beneficio sobre la pérdida de masa ósea que ocurre como consecuencia de la disminución de los niveles de estrógenos durante la menopausia, y que condiciona la aparición de la osteoporosis, un trastorno común en la población a partir de cierta edad, que supone una disminución importante en la calidad de vida de la población que la padece.

La soja es una planta que pertenece al género botánico Glycine y a la familia de las Fabaecae; puede crecer espontánea (Glycine soja Siebold \& Zucc.) o cultivada (Glycine max, Merrill). Es una planta herbácea de cultivo anual, de porte pequeño (unos 40 centímetros aunque en los cultivos pueden llegar a alcanzar los dos metros), tallo erguido, hoja trifoliada, y flores de color blanco o violáceo dispuestas en ramilletes que nacen de la base de las hojas. Su fruto, en vaina, contiene en su interior entre una y cuatro semillas de colores variados. La soja está compuesta químicamente por hidratos de carbono $(23,5 \%)$, proteínas de alto valor biológico (suponen el $37 \%$ de su composición aproximadamente), lípidos (2-3\% de fosfolípidos, especialmente lecitina y ácidos grasos poliinsaturados) y fibra (12\%). Contiene también saponósidos, vitaminas y minerales ( $\mathrm{P}, \mathrm{Ca}, \mathrm{Mg}, \mathrm{Fe})$, enzimas, ácido fítico e isoflavonas (que son las sustancias que le confieren sus características especiales) (1).

\section{FITOESTRÓGENOS E ISOFLAVONAS}

Son compuestos orgánicos producidos por una gran variedad de plantas y que pueden localizarse en distintas partes de las mismas, como las semillas, tallos, raíces o flores; por ello se encuentren ampliamente distribuidos en nuestra alimentación. En las plantas estas sustancias realizan una función protectora frente a una invasión destructiva por microorganismos. Podemos clasificar los fitoestrógenos en dos grandes grupos según su estructura química: por un lado tenemos los lignanos, sintetizados endógenamente en el intestino por acción de la microflora sobre precursores de origen vegetal; y por otro lado los isoflavonoides que son sintetizados exclusivamente por las plantas y poseen actividad estrogénica intrínseca. Existen dos tipos de isoflavonoides: las isoflavonas y los cumestanos. También existe otro grupo de compuestos con actividad estrogénica denominados micoestrógenos, que como hace referencia su nombre, son producidos por determinados hongos. En los isoflavonoides es clave la posición del anillo fenólico en el carbono 3 del heterociclo para la actividad estrogénica (2).

Las isoflavonas y sus derivados glicosídicos son los más abundantes en la naturaleza, habiendo sido identificados 70 moléculas y más de 40 de sus derivados. Se encuentran en plantas de la familia de las leguminosas principalmente y en mayor cantidad, especialmente en las semillas de soja, pero también las contienen plantas de las familias de las solanáceas, gramíneas y rosáceas. Las más conocidas por su actividad estrogénica son la daidzeína y la genisteína. Las isoflavonas no se encuentran en la lecitina de soja, sino que es necesario extraerlas de la semilla de soja con solventes adecuados para respetar la integridad de sus propiedades. Estas sustancias son fenoles heterocíclicos que presentan una gran semejanza estructural con el $17 \beta$-estradiol, el principal estrógeno endógeno. Los derivados glicosídicos presentan una molécula de glucosa o un grupo metilo unido a uno de los grupos funcionales hidroxilo de la molécula principal.

En el caso de precursores, la hidrólisis, que puede ser o no enzimática, se produce en el aparato digestivo por la microflora intestinal; este hecho es nuy importante en la ingesta de fitoestrógenos, pues la microflora, los antibióticos, los prebióticos (entre otros) son los responsables de los distintos niveles de isoflavonas disponibles para ser posteriormente absorbidas. En el intestino delgado los fitoestrógenos requieren el efecto emulsionante de las sales biliares para ser absorbidos e incorporados a la circulación enterohepática y excretados nuevamente con la bilis en forma conjugada; allí son sometidos a la acción de la flora intestinal, reabsorbidos, metabolizados por el hígado y excretados finalmente por la orina. Solamente 
se han encontrado pequeñas cantidades de daidzeína y genisteína en heces, debido probablemente a que han sido químicamente modificadas hacia compuestos conjugados no reconocidos por la microflora del intestino terminal $(1,2)$.

Las isoflavonas estrogénicamente activas se localizan casi exclusivamente en las semillas de las leguminosas y en aquellos productos que las contienen parcialmente o en su totalidad. Sin embargo estas isoflavonas se encuentran de forma mucho más abundante en las semillas de soja y en sus productos derivados, variando la cantidad de las mismas en función del tipo de derivado; este hecho no es conocido por la población general puesto que no está regulado en los etiquetados, lo que es perjudicial para el consumidor, pues uno de los productos más vendidos, la salsa de soja, es precisamente la más pobre en estos compuestos. Por otra parte durante la germinación de las semillas se produce una disminución de las isoflavonas y un aumento del contenido en cumestanos (2).

Los lignanos se encuentran fundamentalmente en cereales, frutas y vegetales, siendo la linaza o aceite de semillas de lino la fuente más rica. También podemos encontrar fitoestrógenos en algunas bebidas alcohólicas procedentes de fermentación de cereales y uvas, sin embargo su concentración es variable en función de las variedades de materia prima y de las técnicas utilizadas para la elaboración.

Por todo ello, el contenido específico de fitoestrógenos en la dieta varía con el tipo de planta o variedad genética (en la soja), el lugar geográfico, la estación del año o el procesado industrial por el cual se ha obtenido el producto alimenticio (2).

\section{CONSUMOS RECOMENDADOS DE SOJA}

Respecto a la cantidad recomendada para el consumo de soja, existen controversias en la literatura. Por una parte habría que tener en cuenta el hecho de que no se conoce exactamente los beneficios que proceden de su actividad hormonal, además el efecto estrogénico que pueda tener es un efecto muy suave, algo que no beneficia a todo el mundo ni a todas las edades.

La menopausia es uno de los momentos donde está más indicado su consumo y siempre bajo prescripción de un médico o especialista, si se va a tomar como suplemento en cápsulas o comprimidos de isoflavonas. Podría estar recomendado (o por lo menos algunos investigadores así lo sugieren) tomar el equivalente a una ración de alimentos de soja (Tabla I); todo esto basándose en los beneficios que aporta la misma a las sociedades que la consumen habitualmente, siendo el ejemplo más típico el sudeste asiático $(3,4)$.

Teniendo en cuenta este último dato, estos alimentos podrían ser considerados como una parte integral del concepto recientemente desarrollado de "alimentos funcionales", que son aquellos alimentos que incluyen un componente que proporciona un beneficio fisiológico específico, además del puramente nutricional; y que suponen una mejora del estado de salud tanto a nivel de la prevención, como en el tratamiento de enfermedades.

Por otra parte, una de las principales preocupaciones sobre el uso de los fitoestrógenos se encuentra en la falta de regulación sobre el control de calidad de las sustancias que están en el mercado a disposición del público, y la falta de información sobre el uso de estas sustancias. Aunque es difícil consumir dosis tóxicas de fitoestrógenos a través del consumo de alimentos naturales, no ocurre lo mismo con el consumo de suplementos a base de concentrados, que pueden facilitar las ingestas elevadas y producir efectos potencialmente peligrosos debido a la sobredosificación (2).

\section{EVIDENCIAS CIENTIIFICAS DE LA RELACIÓN SOJA PREVENCIÓN DE OSTEOPOROSIS}

Los trabajos realizados sobre los beneficios de las isoflavonas de la soja en la prevención de la pérdida de masa ósea relacionada con la menopausia, son muy variados en cuanto a su diseño teniendo en cuenta duración de la suplementación, dosis prescrita y recibida, fuente de soja utilizada, características epidemiológicas de la población, variable de desenlace estudiada, etc.

Algunos estudios sugieren que los efectos de los fitoestrógenos de la soja sobre la densidad mineral ósea son mayores en aquellas mujeres con poca o ninguna producción estrogénica, en mujeres con menopausia tardía, bajo peso, bajo nivel de calcio en el organismo y aquellas con una masa ósea menor; es decir, en aquellas mujeres con una situación menopausiaosteoporosis más deteriorada. Esto ha sido demostrado por el grupo de Chen YM $(5,6)$

Como mencionabamos previamente los diseños son muy variados, de este modo Harkness y cols. (7), evaluaron a 19 muje-

\section{TABLA I}

\begin{tabular}{|c|c|c|c|c|c|c|}
\hline \multicolumn{7}{|c|}{ TABLA I } \\
\hline \multicolumn{7}{|c|}{ FUENTES DE SOJA } \\
\hline Nutrientes & $\begin{array}{l}\text { Miso } \\
100 \mathrm{~g}\end{array}$ & $\begin{array}{l}\text { Lecitina de } \\
\text { soja } 100 \mathrm{~g}\end{array}$ & $\begin{array}{c}\text { Harina } \\
\text { (desaceitada) } \\
100 \mathrm{~g}\end{array}$ & $\begin{array}{l}\text { Salsa de soja } \\
\qquad 100 \mathrm{~g}\end{array}$ & $\begin{array}{l}\text { Tempeh } \\
100 \mathrm{~g}\end{array}$ & $\begin{array}{c}\text { Tofu } \\
\text { (crudo) } \\
100 \mathrm{~g}\end{array}$ \\
\hline Energía (Kcal) & 206 & 763 & 329 & 60 & 199 & 76 \\
\hline Proteínas (g) & 11,8 & - & 47 & 10,5 & 18,9 & 8,1 \\
\hline Lípidos (g) & 6,1 & 100 & 1,2 & 0,1 & 7,7 & 4,8 \\
\hline Poliinsaturados (g) & 3,4 & 45,3 & 0,5 & 0,04 & 4,3 & 2,7 \\
\hline Glúcidos (g) & 28 & - & 38,4 & 5,6 & 17 & 1,9 \\
\hline Fibra (g) & 2,5 & - & 17,5 & 0 & - & 1,2 \\
\hline Calcio (mg) & 66 & - & 241 & 20 & 93 & 105 \\
\hline Hierro (mg) & 2,7 & - & 9,2 & 2,4 & 2,3 & 5,4 \\
\hline
\end{tabular}


res postmenopáusicas a las cuales se suministró un suplemento de isoflavonas durante 6 meses. Encontrando un efecto positivo de la suplementación en la resorción ósea, pero se ignora el tipo de efecto sobre la masa ósea o el riesgo de fracturas. Otro ensayo clínico elaborado por Chiechi y cols. (8) analiza la eficacia de una dieta rica en soja en la prevención de la osteoporosis postmenopáusica, comparándola con la terapia hormonal. Para evaluarlo se reclutaron 187 mujeres postmenopáusicas sin síntomas osteoporóticos, y se compararon tres grupos: uno con tratamiento hormonal, otro con dieta, y un tercer grupo con placebo. Este trabajo concluye que la dieta no es tan efectiva como el tratamiento hormonal para disminuir el recambio óseo, pero que en cambio estimula la actividad osteoblástica. A pesar de estos beneficios, concluyen que deben estudiarse más a fondo los efectos para poder sustituir los tratamientos hormonales. Otro ensayo clínico de dos años de duración llevado a cabo por LydekingOlsen y cols. (9), estudió un grupo de mujeres caucasiacas postmenopáusicas con osteoporosis diagnosticada, o al menos tres factores de riesgo para desarrollarla; se les distribuyó en cuatro grupos de tratamiento: leche de soja con isoflavonas, progesterona subcutánea, leche de soja más progesterona y grupo placebo. Los efectos positivos sobre la masa ósea (medidos en la columna dorsal y la cadera) se encontraron únicamente en los grupos tratados con soja o con hormonas, y sin embargo en el grupo control y en el grupo de combinación de los dos tratamientos, se produjo pérdida de masa ósea, concluyendo que puede ser debido a la interacción negativa entre los tratamientos.

Como podemos comprobar los estudios fueron realizados en mujeres en edad menopausica. No obstante existe un trabajo realizado en varones (10). Este trabajo se realizó en hombres sanos a los cuales se les trató con proteínas de soja, o con leche de soja durante tres meses. Los niveles del IGF-I (factor de crecimiento insulínico) que se asocian con una mayor tasa de formación ósea, aumentaron en los pacientes suplementados con proteínas de soja y no en los suplementados con leche. No se encontraron diferencias entre los grupos en cuanto a marcadores específicos de formación ósea (alcalin-fosfatasa sérica y alcalin-fosfatasa óseo específica), o marcadores específicos de resorción ósea (determinados en orina). Todo esto sugiere que la suplementación con proteínas de soja afecta positivamente a los niveles de IGF-I y puede influir de la misma manera en la masa ósea de los hombres, pero que es necesario elaborar más estudios a largo plazo que investiguen en este sentido.

También se han realizado trabajos de intervención en mujeres jóvenes. De este modo el trabajo de Zitterman y cols. (11) muestra en 14 mujeres jóvenes un aumento de la masa ósea y disminución del turnover óseo al ser suplementadas con isoflavonas. Incluso el grupo de Katsuyama y cols. (12) han demostrado un efecto dosis respuesta en mujeres premenopáusicas, siendo superior el aumento de la formación ósea en las mujeres que consumían suplementos de soja tres veces a la semana, que las que consumían una vez a la semana o una vez al mes.

A pesar de los resultados favorables observados en los estudios previamente mencionados, también existen trabajos donde no se han detectado efectos beneficiosos. Estos estudios son el ensayo clínico doble ciego de Gallagher y cols. (13) en el que se estudiaron 65 mujeres durante 15 meses, repartidas en dos grupos a los cuales se administraban diferentes dosis de proteínas de soja con isoflavonas, y un tercer grupo con proteínas de soja libres de isoflavonas. Los niveles en sangre de estos compuestos dependían de la dosis administrada, pero no se observó un aumento de la densidad mineral ósea medida en la espina dorsal y el cuello del fémur; sin embargo aumentó significativamente la densidad mineral ósea a nivel de trocánter en los pacientes con placebo, sin encontrar tampoco se encontraron efectos significativos en el metabolismo lipídico. El otro trabajo con resultados negativos es el ensayo clínico de Dalais y cols. (14) en el cual se trataron a 106 mujeres postmenopáusicas divididas en dos grupos: uno con suplementación con proteínas de soja y otro placebo (sin suplementación). Los resultados fueron favorables en los niveles lipídicos (disminución de los triglicéridos, mantenimiento de los niveles de HDL) pero no se observaron efectos estrogénicos ni cambios en los marcadores de resorción ósea. Todo esto sugiere que los mecanismos por los cuales actúa la soja son diferentes a los mecanismos hormonales. Incluso existen trabajos en modelos animales que han demostrado una mejoría en la microestructura ósea tras la suplementación con soja sin conseguir aumentar la masa ósea (15).

En conclusión, la mayoría de los estudios evaluados concluyen que los efectos positivos que se observan no están del todo claros, por ser trabajos con poblaciones de estudio pequeñas, o de corta duración. No obstante se puede concluir que es necesaria más investigación al respecto, aunque pueden ser válidos como tratamientos preventivos. Por tanto la dieta equilibrada como mecanismo de prevención y tratamiento debe ser utilizada como primer escalón terapéutico en los pacientes con riesgo de osteoporosis, incluso la suplementación de esta dieta con soja puede presentar beneficios adicionales, sobre todo en grupos de alto riesgo, con unos riesgos para la salud mínimos.

D. A. DE LUIS, J. L. PÉREZ CASTRILLÓN, R. ALLER, J. CULEBRAS ${ }^{1}$

Instituto de Endocrinología y Nutrición. Facultad de Medicina. Unidad de Apoyo a la Investigación. Hospital Universitario Río Hortega. Valladolid. ${ }^{\text {SServicio de }}$ Cirugía General. Complejo Hospitalario de Leon. Léon 


\section{Bibliografía}

1. Tomé D, Mariotti F. La soja en la alimentación. Alimentación, Nutrición y Salud 2000; 7: 31-33.

2. Becerra Fernández A. La edad de la menopausia. 2003. Ed.: Díaz de Santos. Madrid.

3. Adlercreutz H, Mazur W. Phyto-estrogen and western diseases. Ann Med 1997; 29: 95-1201.

4. Chen Z, Zheng W, Custer LJ, et al. Ususal dietary consumptiom of soy foods and its correlation with the excretion rate of isoflavonoids in overnight urine samoles among Chinese women in Shanghai. Nutr Cancer 1999; 33: 82-87.

5. Chen YM, Ho SC, Lam SS, Ho SS, Woo JL. Beneficial effect of soy isoflavones on bone mineral content was modified by years since menopause, body weight, and calcium intake: a double-blind, randomized, controlled trial. Menopause 2004; 11: 246-254.

6. Chen YM, Ho SC, Lam SS, Ho SS, Woo JL. Soy isoflavones have a favorable effect on bone loss in Chinese postmenopausal women with lower bone mass: a double-blind, randomized, controlled trial. J Clin Endocr Metab 2003; 88: 47410-4747.

7. Harkness LS, Fiedles K, Sehgal AR, Oravec D, Lerner E. Decreased bone resorption with soy isoflavone supplementation in postmenopausal women. J WOmens Health 2004: 13: 1000-1007.

8. Chiechi LM, Secreto G, D'Amore M, Fanelli M, Venturelli E, Cantatore F, et al. Efficacy of a soy rich diet in preventing postmenopausal osteoporosis: the Menfis randomized trial. Maturitas 2002: 42: 295300 .
9. Lydeking-Olsen E, Beck-Jensen JE, Setchell KD, Hola-Jensen T. Soy milk or progesterone for prevention of bone loss - a 2 year randomized, placebo-controlled trial. Eur J Nutr 2004; 43: 246-257.

10. Khalil DA, Lucas EA, Juma S, Smith BJ, Payton ME, Arjmandi BH. Soy protein supplementation increases serum insulin-like growth factor-I in young and old men but does not affect markers of bone metabolism. Eur J Nutr 2002: 132: 2605-2608.

11. Zittermmann A, Geppert J, Baier S, Zehn N, Gouni-Berthold I, Berthold HK, et al. Short term efects of high dsoy supplementation on sex hormones, bone markers, and lipid parameters in young female adults. Eur J Nutr 2004; 43: 100-108.

12. Katsuyama H, Ideguchi S, Fukunaga M, Fukunaga T, Saijoh K, Sunami S. Promotion of bone formation by fermented soybean (NATTO) intake in premenopuasl women. J Nutr Sco Vitaminol 2004; 50: 114-120.

13. Gallagher JC, Satpathy R, Rafferty K, Haynatzka V. The effect of soy protein isolate on bone metabolism. Menopause 2004: 11: 290-298.

14. Dalais FS, Ebeling PR. Kotsopoulos D, McGrath BP, Teede HJ. The effects of soy protein containing isoflavones on lipids and indices of bone resorption in postmenopausal women. Clin Endocr 2003: 58: 704709.

15. Devareddy L, Khalil DA, Smith BJ, Lucas EA, Sound do Y, AMrlow $\mathrm{DD}$, et al. Soy moderately improves microstructurak properties without affecting bone mass in an ovariectomized rat model of osteoporosis. Bone 2006; 38: 686-693. 